\title{
Avalanche boron fusion by laser picosecond block ignition with magnetic trapping for clean and economic reactor
}

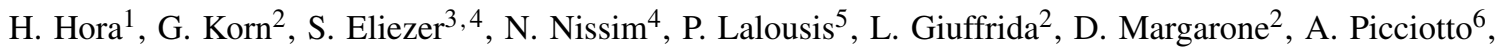 \\ G.H. Miley ${ }^{7}$, S. Moustaizis ${ }^{8}$, J.-M. Martinez-Val ${ }^{3}$, C.P.J. Barty ${ }^{9}$, and G.J. Kirchhoff ${ }^{10}$ \\ ${ }^{1}$ Department of Theoretical Physics, University of New South Wales, Sydney 2052, Australia \\ ${ }^{2}$ ELI-Beamline Project, Inst. Physics, ASCR, PALS Center, Prague, Czech Republic \\ ${ }^{3}$ Institute of Nuclear Fusion, Polytechnic University of Madrid, Madrid, Spain \\ ${ }^{4}$ Soreq Research Center, Yavne, Israel \\ ${ }^{5}$ Institute of Electronic Structure and Lasers FORTH, Heraklion, Greece \\ ${ }^{6}$ Micro-Nano Facility, Fondazione Bruno Kessler, 38123 Trento, Italy \\ ${ }^{7}$ Department of Nuclear Plasma \& Radiological Engineering University of Illinois, Urbana, IL, USA \\ ${ }^{8}$ Technical University Crete, Laboratory of Matter Structure and Laser Physics, Chania, Greece \\ ${ }^{9}$ Lawrence Livermore National Laboratory, Livermore, CA, USA \\ ${ }^{10}$ UJK Management GmbH, 85586 Poing, Germany \\ (Received 12 February 2016; revised 11 July 2016; accepted 19 July 2016)
}

\begin{abstract}
Measured highly elevated gains of proton-boron (HB11) fusion (Picciotto et al., Phys. Rev. X 4, 031030 (2014)) confirmed the exceptional avalanche reaction process (Lalousis et al., Laser Part. Beams 32, 409 (2014); Hora et al., Laser Part. Beams 33, 607 (2015)) for the combination of the non-thermal block ignition using ultrahigh intensity laser pulses of picoseconds duration. The ultrahigh acceleration above $10^{20} \mathrm{~cm} \mathrm{~s}^{-2}$ for plasma blocks was theoretically and numerically predicted since 1978 (Hora, Physics of Laser Driven Plasmas (Wiley, 1981), pp. 178 and 179) and measured (Sauerbrey, Phys. Plasmas 3, 4712 (1996)) in exact agreement (Hora et al., Phys. Plasmas 14, 072701 (2007)) when the dominating force was overcoming thermal processes. This is based on Maxwell's stress tensor by the dielectric properties of plasma leading to the nonlinear (ponderomotive) force $f_{\mathrm{NL}}$ resulting in ultra-fast expanding plasma blocks by a dielectric explosion. Combining this with measured ultrahigh magnetic fields and the avalanche process opens an option for an environmentally absolute clean and economic boron fusion power reactor. This is supported also by other experiments with very high HB11 reactions under different conditions (Labaune et al., Nature Commun. 4, 2506 (2013)).
\end{abstract}

Keywords: boron fusion energy; dielectric nonlinear force explosion; economic reactor; environmentally clean energy; picosecond-non-thermal plasma block ignition

\section{Introduction}

Controlled fusion reaction for energy production is so highly attractive that enormous research was invested during the past 60 years focusing on the reaction of heavy and superheavy hydrogen, deuterium $\mathrm{D}$ and tritium $\mathrm{T}$, respectively. Impressive advances were achieved while it is well evident

Correspondence to: H. Hora, Department of Theoretical Physics, University of New South Wales, Sydney 2052, Australia.

Email: h.hora@unsw.edu.au that the aimed power station is still far away in the future. A special problem for DT fusion is that the generation of neutrons apart from the clean helium resulted in the statement (see Nature, citation Butler ${ }^{[1]}$ ) that the magnetic confinement option International Thermonuclear Experimental Reactor (ITER) will result in the 'hottest radioactive working environment on earth'.

The fusion reaction of hydrogen (protons) with ${ }^{11} \mathrm{~B}$ (HB11) initially did not at all show primary neutron generation $^{[2]}$ while further any side reaction produced less 
tolerable radioactivity per produced energy ${ }^{[3]}$. This takes into account the extreme non-equilibrium conditions. The energy generated by the HB11 reaction ${ }^{[3]}$

$$
\mathrm{H}+{ }^{11} \mathrm{~B}=3{ }^{4} \mathrm{He}+8.9 \mathrm{MeV},
$$

was measured even before the DT fusion reaction was discovered. It was early realized that the HB11 reaction is extremely more difficult than DT fusion. When using nanosecond laser pulses for compression, heating and ignition of HB11, densities above 100.000 times of solid are needed ${ }^{[4]}$. Compressing DT to the order of the thousand times of solid state has been verified ${ }^{[5]}$ but the level of more than hundred times higher densities may be impossible.

The enormous difficulties can be overcome by using a nonthermal ignition scheme ${ }^{[6]}$. On top the recent measurement of several kilotesla magnetic fields ${ }^{[7]}$ has to be involved but key ingredient on the way to a boron laser fusion reactor is the experimental discovery ${ }^{[8,9]}$ of the avalanche reaction of HB $11^{[1,10]}$ to open a radical new solution for fusion energy.

\section{Non-thermal plasma block ignition of fusion}

For the following new aspects with boron fusion it took dozens of years to realize the basic difference between the thermodynamic dominated laser fusion with nanosecond pulses in contrast to the entirely different non-thermal processes with the thousand times shorter picosecond laserplasma interaction. The difference is given by the force density $\mathbf{f}$ in the plasma being not only determined by the gas dynamic pressure $p$ but also by the force $\mathbf{f}_{\mathrm{NL}}$ due to electric $\mathbf{E}$ and magnetic $\mathbf{B}$ laser fields of frequency $\omega$,

$$
\mathbf{f}=-\nabla p+\mathbf{f}_{\mathrm{NL}}
$$

where the force $\mathbf{f}_{\mathrm{NL}}$ is given by Maxwell's stress tensor as Lorentz and gauge invariant nonlinear force determined by quadratic terms of fields (Ref. [11]: see Equation (8.88))

$$
\begin{aligned}
\mathbf{f}_{\mathrm{NL}}= & \nabla \bullet\left[\mathbf{E E}+\mathbf{H H}-0.5\left(\mathbf{E}^{2}+\mathbf{H}^{2}\right) \mathbf{1}\right. \\
& \left.+(1+(\partial / \partial t) / \omega)\left(\mathbf{n}^{2}-1\right) \mathbf{E} \mathbf{E}\right] /(4 \pi) \\
& -(\partial / \partial t) \mathbf{E} \times \mathbf{H} /(4 \pi c),
\end{aligned}
$$

where $\mathbf{1}$ is the unity tensor and $\mathbf{n}$ is the complex optical constant of the plasma given by the plasma frequency $\omega_{p}$. At plane laser wave interaction with a plane plasma front, the nonlinear force reduces to

$$
\begin{aligned}
\mathbf{f}_{\mathrm{NL}} & =-(\partial / \partial x)\left(\mathbf{E}^{2}+\mathbf{H}^{2}\right) /(8 \pi) \\
& =-\left(\omega_{p} / \omega\right)^{2}(\partial / \partial x)\left(E_{v}^{2} / \mathbf{n}\right) /(16 \pi),
\end{aligned}
$$

showing how the force density is given by the negative gradient of the electromagnetic laser field energy density including the magnetic laser field from Maxwell's equations.

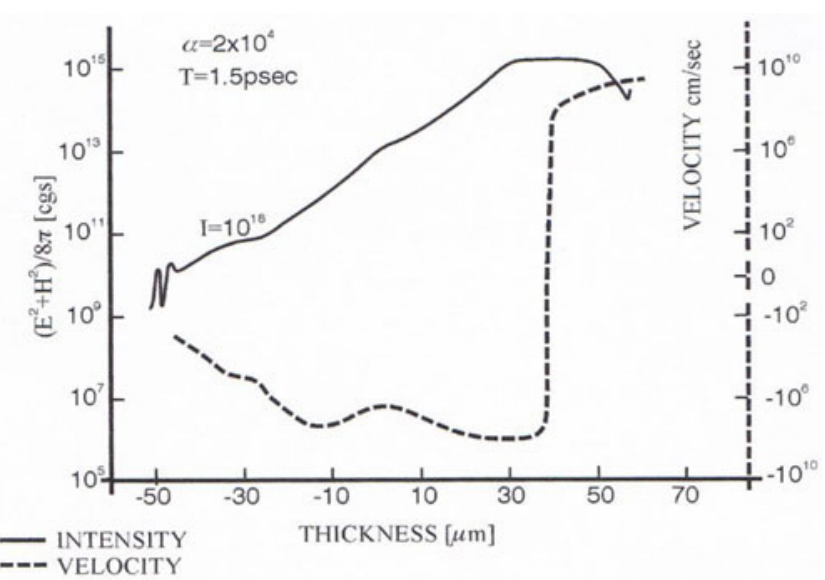

Figure 1. $10^{18} \mathrm{~W} \mathrm{~cm}^{-2}$ neodymium glass laser incident from the right-hand side on an initially $100 \mathrm{eV}$ hot deuterium plasma slab whose initial density has a very low reflecting bi-Rayleigh profile, resulting in a laser energy density and a velocity distribution from plasma hydrodynamic computations at time $t=1.5 \mathrm{ps}$ of interaction. The driving nonlinear force is the negative of the energy density gradient of the laser field $\left(\mathbf{E}^{2}+\mathbf{H}^{2}\right) / 8 \pi$. The dynamic development of temperature and density had accelerated the plasma block of about 15 vacuum wave length thickness of the dielectric enlarged skin layer moving against the laser (positive velocity) and another block into the plasma (negative velocity) showing ultrahigh $>10^{20} \mathrm{~cm} \mathrm{~s}^{-2}$ acceleration (Ref. [12]: Figures 10.18a\&b) as computer result of 1978.

$E_{v}$ is the amplitude of the electric laser field in vacuum after time averaging. The second expression in Equation (4) is Kelvin's formulation of the ponderomotive force in electrostatics of 1845 .

The difference to laser interaction by nanosecond thermal interaction against picosecond non-thermal nonlinear force driving is determined by $\mathbf{f}_{\mathrm{NL}}$ interaction dominating in Equation (2). For the nanosecond interaction, the first term in Equation (2) dominates at low laser intensities while with picosecond, the second term can dominate in which case the laser intensity has to be high enough that the quiver energy of the electrons of the laser field is higher than their thermal energy of motion. A numerical example about nonlinear force acceleration of a slab of deuterium plasma irradiated by a neodymium glass laser pulse of $10^{18} \mathrm{~W} \mathrm{~cm}^{-2}$ intensity is in Figure 1. During the $1.5 \mathrm{ps}$, the plasma reached velocities above $10^{9} \mathrm{~cm} \mathrm{~s}^{-1}$ showing the ultrahigh acceleration above $10^{20} \mathrm{~cm} \mathrm{~s}^{-2}$. The generation of the plasma blocks, one moving against the laser light and the other into the higher density target is the result of a non-thermal collisionless absorption and should not be understood as radiation pressure acceleration but as a dielectric explosion driving the plasma blocks.

The experimental proof of the ultrahigh acceleration was possible ${ }^{[13]}$ in full agreement with the results of computations in 1978 with domination by the nonlinear force, when laser pulses of higher than terawatt power and about ps duration were available after discovering the chirped pulse amplification $(\mathrm{CPA})^{[14,15]}$. With these picosecond ultrahigh accelerations, the plasma block ignition of solid density 
DT by the nonlinear force was possible and updated ${ }^{[16]}$. Computation of DT fusion using the ps-block ignition ${ }^{[17]}$ showed many details of the generated fusion flames with velocities of few $1000 \mathrm{~km} \mathrm{~s}^{-1}$, the delayed generation of a Rankine-Hugoniot shock front, local distribution of reaction rates, etc., however, only in one-dimensional plane wave computations.

To summarize, what is experimentally and theoretically clarified is the ultrahigh acceleration of plasma blocks measured by Sauerbrey ${ }^{[13]}$, repeated by Földes et al. ${ }^{[18]}$, and measured by Badziak et al. ${ }^{[19]}$ as skin layer plasma block moving against the laser light all in exact agreement with the numerical prediction (Figure 1) based on non-thermal nonlinear force processes ${ }^{[20-22]}$ as dielectric explosion. The necessary conditions are unique: providing a flat interaction surface, avoiding self-focusing, and providing an extremely high contrast of the laser pulse. The exclusion of relativistic self-focusing was measured in a most exceptional way by Zhang et al. ${ }^{[23]}$ in contrast to the very broad stream of measurements where always very intense and hard $\mathrm{x}$-ray emission was measured. The conditions of flat interaction and extremely high contrast arrived at low intensity soft $\mathrm{x}$-ray emission with the discovery that this was due to the elimination of self-focusing as the key condition for the experiments $^{[13,18,19]}$. This is a perfect solidification of the picosecond nonlinear force non-thermal block acceleration process.

The broad stream of experiments did not fulfill these conditions beginning with the use of not sufficiently clean laser pulses ${ }^{[24]}$ and most of the phenomena reported from numerous experiments with not fulfilling the conditions e.g., Ref. [25]. For the following studies it is important to measure the variations of the conditions to re-establish what is needed for using the fulfilled conditions of the successful experiments ${ }^{[13,18,19,23]}$. After realizing this knowledge the very diversifying acceleration mechanisms can be categorized experimentally as shown in recent examples ${ }^{[26-28]}$.

For the theoretical-numerical understanding it should be noticed how extensive particles-in-cell (PIC) computations $^{[29]}$ arrived at the same acceleration of plasma blocks in the $70 \mathrm{MJ}$ ion energy range as by hydrodynamic computations $^{[30,31]}$.

\section{Radical change for boron fusion}

The use of the confirmed ultrahigh acceleration of plasma blocks for igniting a fusion reaction ${ }^{[16,17]}$ was based on the computational work of $\mathrm{Chu}^{[32]}$ and of Bobin ${ }^{[33]}$. For initiating the ignition of a solid density DT target by a plane geometry laser pulse, it was numerically evaluated that the laser pulse had to be short in the range of $1 \mathrm{ps}$ and the energy flux density $E^{*}$ had to be above the threshold $E_{o}{ }^{*}$

$$
E^{*}>E_{\mathrm{o}}{ }^{*}=5 \times 10^{8} \mathrm{~J} \mathrm{~cm}^{-2} \text {. }
$$

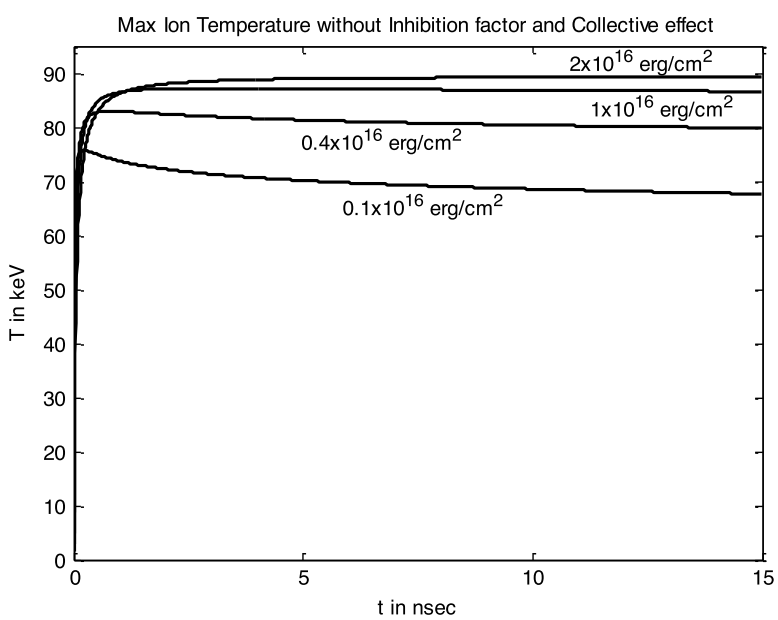

Figure 2. The picosecond laser pulse initiation of fusion flame in solid density HB11: maximum temperature of fusion reaction at time after initiation showing ignition at laser energy flux of $8 \times 10^{8} \mathrm{~J} \mathrm{~cm}^{-2[6]}$.

These conditions were far beyond the lasers of that time. This was the main reason to work with laser pulses of nanosecond duration and to use the thermal processes of ablation, compression, and thermonuclear ignition for generating fusion power. These conditions changed with the discovery of $\mathrm{CPA}^{[14,15]}$ and the updating of the computations of the fusion reactions based on the same hydrodynamic conditions as ${ }^{[32]}$ arrived at the same result of Equation (5) for DT fusion ${ }^{[34]}$. Updating by using collective effects and inhibition of thermal conductivity at inhomogeneous plasmas resulted in a reduction of the threshold $E_{\mathrm{o}}{ }^{*}$ to lower values up to a factor $20^{[34]}$. These results were confirmed ${ }^{[18]}$ with deriving a number of further details by using a basically different genuine multi-fluid hydrodynamic code ${ }^{[35,36]}$.

Irradiating the picosecond pulses on solid density DT fuel for initiating a fusion reaction in plane geometry ${ }^{[32]}$ needed a threshold for the energy flux density of $E^{*}=$ $5 \times 10^{8} \mathrm{~J} \mathrm{~cm}^{-2}$, Equation (5). This non-thermal picosecond ignition of fusion as described in the preceding section, was exactly reproduced by a similar one fluid computation, where however later discovered plasma properties as the inhibition factor and the collective collisions had to be included and led to the updating for $\mathrm{DT}^{[33]}$. When instead of the DT crosssections, those for the much more difficult case for HB11 were used, in the same computations, a most unexpected and surprising result was achieved. Instead of the earlier known extremely more difficult ignition compared with DT, the threshold $E^{*}$ for HB11 was of nearly the same value ${ }^{[6,16,37]}$. This can be seen in Figure 2 where in the same way as calculated before by $\mathrm{Chu}^{[32]}$, the maximum temperature of the reacting plasma depending on the time $t$ after the ps long initiation process was calculated.

In difference to the computations with the single fluid hydrodynamics ${ }^{[6,16,34,37]}$, when performing the computations with the much more detailed genuine two-fluid hydrodynamics $^{[35,36]}$, the properties of the Rankine-Hugoniot 
shock generation were reproduced for HB11 in a more general way showing the plasma collision generated broadening of the shock front, the very high internal electric fields and their decay, and their delayed built-up within hundreds of picoseconds after the picosecond laser pulse initiation ${ }^{[17]}$.

The difference between DT and HB11 was the reaction temperature, which was as expected above the threshold with respect to losses by bremsstrahlung emission above the limiting temperatures of $4 \mathrm{keV}$ for DT and $65 \mathrm{keV}$ for HB11. This was automatically included since the initial computations by $\mathrm{Chu}^{[32]}$.

\section{Cylindrical plasma trapping by ultrahigh laser gener- ated magnetic fields}

Despite of the attention given to the result ${ }^{[6,37]}$ by finding the nonlinear force conditions for abolishing the difference of laser fusion between HB11 and DT, this result was limited to cases of plane geometry. In reality the laser pulses are not infinitely spread by beams and the radial losses at target interaction and radiation emission have to be taken into account. The first step was to use spherical geometry. In this case, the computations profit from a compression of the initially solid-state fusion fuel. The chirped pulse amplification for picosecond laser pulses up to exawatt power can be considered possible in further future ${ }^{[16,38]}$. However with these pulses, the gains both for DT or for HB can be only up to the range of few hundreds with exawatt laser pulses. And this is too low though 200-PW-picosecond laser pulses are available next ${ }^{[39]}$.

A way other than spherical geometry was possible with cylindrical geometry after the discovery of Fujioka et al. ${ }^{[7]}$ to generate magnetic fields of $4.5 \mathrm{kT}$ by laser interaction shown in Figure 3. Hydrodynamic computations demonstrate how a cylindrical fusion plasma in solid-state density of HB11 coaxially located within the coils in Figure 3 can be trapped within the volume of few cubic millimeters with a magnetic field of $10 \mathrm{kT}$. The fusion reactions for HB11 show ignition by using binary reactions during few nanoseconds of the presence of the ultrahigh magnetic field from the generated fluid of alpha particles being in a similar way trapped and cylindrically confined using then the genuine two-fluid plasma hydrodynamics ${ }^{[10,17,35,36,38]}$.

Extensive hydrodynamic computations for $10 \mathrm{kT}$ magnetic trapping in support of the results in Ref. [10] are collected in Ref. [40] as figures, where 10.13 to 10.23 for laser pulses of picosecond duration and $10^{20} \mathrm{~W} \mathrm{~cm} \mathrm{~cm}^{-2}$ for laser intensity by block ignition as described in the preceding section. The cylindrical trapping of the laser pulse within the coils and their very slow radial expansion against the magnetic field of $10 \mathrm{kT}$ was shown in very detailed calculations, e.g., if the coaxially located solid HB11 target was of $1 \mathrm{~cm}$ length and $1 \mathrm{~mm}$ radius.

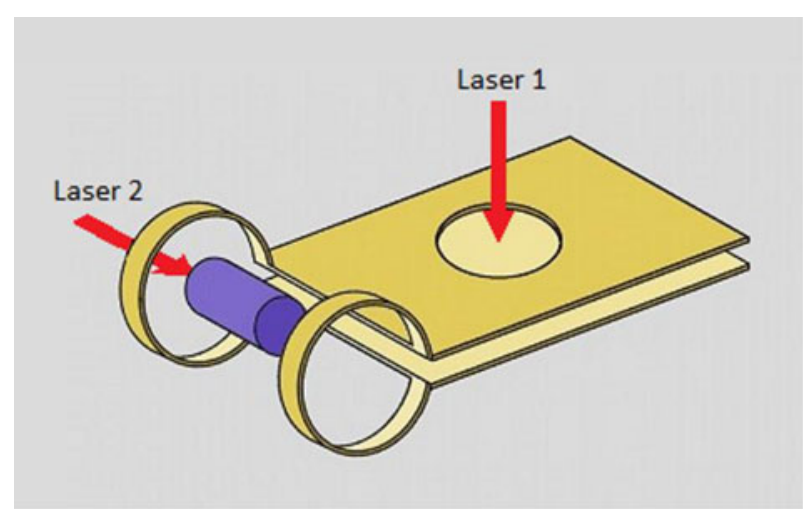

Figure 3. Generation of a $4.5 \mathrm{kT}$ magnetic field within the coils of about 2 ns duration in the coils by firing a $>$ kilojoule nanosecond laser pulse 1 into the hole between the plates ${ }^{[7]}$.

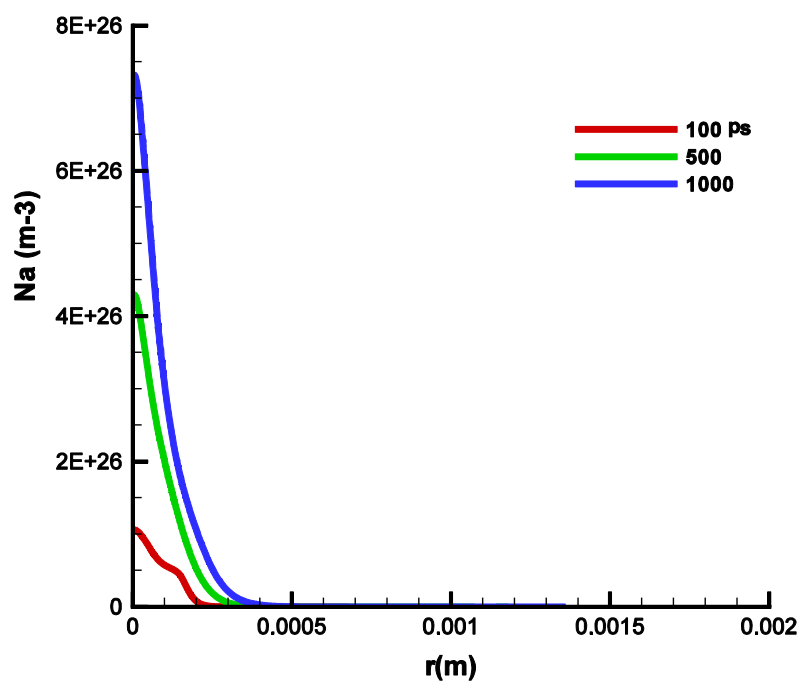

Figure 4. Alpha density $N_{a}$ depending on the radius $r$ at different times (from lowest to highest curves for 100,500 and $1000 \mathrm{ps}$, respectively) showing ignition from the increase of the curves on time calculated for irradiating a cm long solid HB11 cylinder of $0.2 \mathrm{~mm}$ diameter with a picosecond laser pulse of $30 \mathrm{PW}$ for plasma block initiation of the fusion reaction $^{[10]}$.

Complete trapping beyond nanosecond reaction time was confirmed while the propagation of the reaction front parallel to the magnetic field was of few thousand $\mathrm{km} \mathrm{s}^{-1}$ and was well comparable to cases with DT fusion at the same fusion scheme. For testing the radial trapping by the ultrahigh magnetic fields ${ }^{[7]}$, an even thinner fuel cylinder of $0.2 \mathrm{~mm}$ was used $^{[10]}$. Then a slow expansion of the alpha particle fluid - well at more than 100 times lower density than the solid-state fuel - is shown for different times after the picosecond plasma block ignition with a $30 \mathrm{PW}$ laser pulse in Figure $4^{[10]}$. Block ignition of an HB11 fusion fuel cylinder of $1 \mathrm{~mm}$ radius in the axis of the coil by a picosecond laser pulse 2 needs powers above exawatt for gains in the range of 300. This is a next step of developing highest power laser pulses $^{[39]}$. 


\section{Breakthrough based on HB11 avalanche reactions after measuring elevated fusion gains}

All hydrodynamic computations reported up to this stage were using only binary reactions for HB11 fusion in the same way as given for DT. Higher reaction gains were in difference to DT considered by the fact that the generated alpha particles with energies of $2.9 \mathrm{MeV}$ from HB11 can transfer energies around $600 \mathrm{keV}$ to boron nuclei by elastic collisions. As it is well known as an anomaly compared with all fusion reactions, the fusion cross-section for HB11 is about ten times higher for HB11 at $600 \mathrm{keV}$ center-ofmass energy, resulting in a secondary reaction with protons producing three new alphas with a subsequent possibility of an avalanche reaction. Discussions were documented since the 2012 IAEA fusion conference ${ }^{[41]}$ and were used as preliminary estimations ${ }^{[10]}$ on which an international patent application was based.

Measurements of alpha particles from HB11 reactions at irradiation with picosecond laser pulses were reported first $^{[42]}$ at numbers of about 1000, just above the detection threshold. More than one million alpha particles were detected in an experiment ${ }^{[9]}$ using a combination of laser driven ion acceleration and direct laser interaction with picosecond pulses. About billion alpha particles were measured at straight forward irradiation by pulses of about 100 ps duration and $500 \mathrm{~J}$ energy from the iodine Prague Asterix Laser System (PALS) laser ${ }^{[8]}$ when using a most exotic target. This can be found in silicon crystals where about $10 \%$ boron was incorporated. Substituting extremely low boron concentrations within the lattice of silicon for $\mathrm{p}$ conductivity was the key process for producing high ultrahigh frequency diodes ${ }^{[43]}$ and later for the crucial discovery of the polar transistor ${ }^{[44]}$. A laser irradiation may be mentioned how an anomalous photoemission could be measured of these semiconductors producing electric double layers by surface traps ${ }^{[45]}$ including boron doped silicon. The interest in semiconductor physics for transistor effects was studying higher and higher boron concentrations up to densities with states of degenerate holes for p-conducting.

Just this extremely high boron doping for studying semiconducting properties of silicon led to the combination for laser-fusion experiments at PALS ${ }^{[8]}$ with the most surprising measurement of the billion fusion reactions. These reaction gains were of extremely elevated values. Initially, these results were not referring to the relation of an avalanche reaction process ${ }^{[8]}$. However when these experiments ${ }^{[46,47]}$ were evaluated as a result of several orders of magnitudes higher than expected from binary reactions - up to gains even higher than from DT by using reasonable comparisons - it was then evident, that there was the avalanche reaction happening ${ }^{[1]}$.

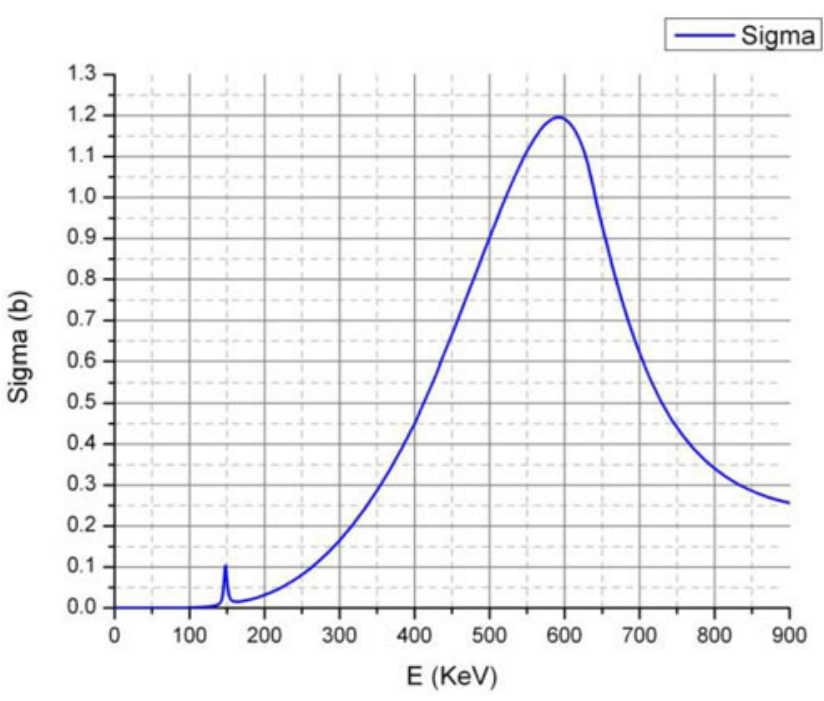

Figure 5. The proton-boron 11 fusion cross-section $\sigma$ recalculated from Nevins and Swain ${ }^{[48,49]}$.

This proof of the avalanche process could then be taken as a reality for the anticipated evaluation ${ }^{[10]}$ of very high reaction gains to be used for the $>$ PW-ps laser pulse plasma block generation for initiation of the non-thermal ignition of the HB11 reactions in the cylindrical volumes of solid density fusion fuel at trapping by the ultrahigh $10 \mathrm{kT}$ magnetic fields described in the preceding section. The generation of more than gigajoule energy of alpha particles by irradiating $30 \mathrm{~kJ}$ laser pulses can then be a way for designing a power reactor. The process during about one nanosecond within a volume of few cubic millimeters gives evidence that this can be used only for a controlled reaction for generating electricity.

The avalanche process can be explained on the elastic central collision where an initially resting ${ }^{11} \mathrm{~B}$ or proton nucleus of mass $m_{2}$ gains energy from the energy $E_{\alpha}$ of an alpha particle of mass $m_{1}$. After an alpha with an energy $E_{\alpha}=2900 \mathrm{keV}$ has its second collision with a proton and this proton collides with a boron11, one gets an energy in their center-of-mass system of reference and energy $E_{\mathrm{cm}}\left(p B^{11}\right)^{[48,49]}$, Figure 5.

$$
E_{\mathrm{cm}}\left(p B^{11}\right)=\left(\frac{11}{12}\right)\left(\frac{16}{25}\right)\left(\frac{9}{25}\right) E_{\alpha}=612.5[\mathrm{keV}]
$$

This energy is within the maximum cross-section $\sigma_{\max }$ of HB $11^{[48,49]}$ as is shown in Figure 5. We get the energy for $\mathrm{HB}^{11}$ maximum cross-section $\sigma$ from the alpha's collisions with protons (that then collides with $\mathrm{B}^{11}$ ) to get the fusion, as the avalanche mechanism because of the multiplication through generation of three secondary alpha particles.

In this process we get 2 classes of proton densities, $n_{p 1}$ that did not have any alpha collision and $n_{p 2}$ that collided with alpha and got the right energy to have a proton-boron 11 
collision at maximum nuclear cross-section. It is conceivable to assume for this experiment ${ }^{[8]} n_{p}=n_{p 1}+n_{p 2}$ and $n_{p 1} \gg$ $n_{p 2}=n_{\alpha}$ yielding the rate equation for the alpha particles

$$
\frac{\mathrm{d} n_{\alpha}}{\mathrm{d} t} \approx 3 n_{p} n_{B}\langle\sigma v\rangle+3 n_{\alpha} n_{B} \sigma_{\max } u .
$$

The second term is caused by the protons that collided with the alphas while the first term in this equation is caused by protons created in the laser-plasma interaction and are returned back into the target by the inverted double layer simulations ${ }^{[36]}$. Taking the data from the PALS experiment ${ }^{[8]}$ Equation (7) can be solved numerically. In particular, the proton energy distribution as given in this experiment can be written as $\mathrm{d} N_{p} / \mathrm{d} E=N_{0}\left[\mathrm{MeV}^{-1}\right]$ for $0<E<1 \mathrm{MeV}$ and $\mathrm{d} N_{p} / \mathrm{d} E=0$ for $E>1 \mathrm{MeV}$, where $N_{p}$ is the proton volume integrated density number and $N_{0}=$ $10^{11}$ is the total number of protons under consideration. This distribution implies

$$
\begin{aligned}
& \frac{\langle\sigma v\rangle}{\sigma_{\max } u}=\frac{\int_{0}^{\infty} f(E) \sigma(E) E^{1 / 2} \mathrm{~d} E / \int_{0}^{\infty} f(E) \mathrm{d} E}{(1.2 \text { barn }) \sqrt{0.6 \mathrm{MeV}}} \approx 0.2, \\
& f(E)= \begin{cases}N_{0}=10^{11}\left[\mathrm{MeV}^{-1}\right] & \text { for } 0<E<1 \mathrm{MeV} \\
0 & \text { for } 1 \mathrm{MeV}<E .\end{cases}
\end{aligned}
$$

Therefore to a good approximation we get the following solution

$$
\begin{aligned}
N_{\alpha} & =\frac{\langle\sigma v\rangle}{\sigma_{\max } u} N_{p}\left(\mathrm{e}^{\tau / \tau_{A}}-1\right) \approx 0.2 N_{0}\left(\frac{\tau}{\tau_{A}}\right), \\
\tau_{A} & \equiv \frac{1}{3 n_{B} \sigma_{\max } u},
\end{aligned}
$$

where $N_{0}$ is of the order of few times $10^{11}$ and $N_{\alpha}$ of the order of $10^{9}$ are accordingly the volume integrated density numbers as given in the measurement ${ }^{[8]} \cdot \tau_{A}$ is defined as the avalanche time and the interaction time $\tau$ to create alphas. In the Prague experiment $\tau_{A}$ is of the order of $100 \mathrm{~ns}\left(n_{B}=\right.$ $10^{22} \mathrm{~cm}^{-3}, \sigma_{\max }=1.2$ barns and $u=10^{9} \mathrm{~cm} \mathrm{~s}^{-1}$ ) which means that alphas are created during the range of $1 \mathrm{~ns}$.

The HB11 plasma contains then a component of the lower density of a fluid of alpha particles with the elastic collisions of boron nuclei at around $600 \mathrm{keV}$ energy. This is a typical non-ideal plasma known from other applications ${ }^{[50]}$.

A completely different approach to study the avalanche reaction (alternatively called chain reaction $)^{[9,51]}$ uses the experiment of Refs. [9, 47] but under more general conditions including natural boron containing also the isotope ${ }^{10} \mathrm{~B}$. The side reaction of the alphas with ${ }^{11} \mathrm{~B}$ is to produce ${ }^{14} \mathrm{C}$ resulting neutrons carrying $5 \times 10^{-6}$ of the energy of the alphas of Equation (1).

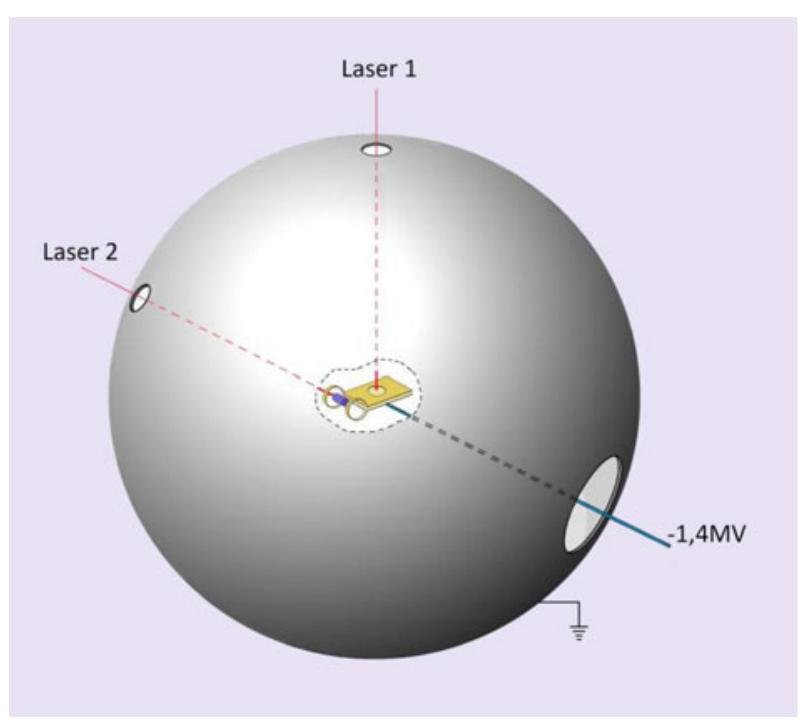

Figure 6. Scheme of an HB11 fusion reactor without radioactive radiation problems is based on non-thermal plasma block ignition by nonlinear forces (Section 2) by a 30-kJ-picosecond laser pulse 2 (Figure 2) where the solid hydrogen-boron fuel in the cylindrical axis of the magnetic coil is trapped by a $10 \mathrm{kT}$ field sustained for about $1 \mathrm{~ns}$ after being generated by a nanosecond long laser pulse 1. The central reaction unit (Figure 3 in the center of the sphere) is electric charged to the level of $-1.4 \mathrm{MV}$ against the wall of a sphere producing alpha particles (helium nuclei) of more than a gigajoule energy, of which a small part is needed for the operation of the laser pulses. One part of the gained costs of electricity is needed for the apparatus of the central reaction and for the boron metal of the fuel being destroyed at each reaction ${ }^{[1,10,52,53]}$.

\section{Clean fusion power reactor}

After the experimental confirmation ${ }^{[1]}$ of the avalanche ignition of the HB11 picosecond plasma block initiation of the fusion reaction with high gains at trapping by ultrahigh magnetic fields and the modeling of the avalanche process ${ }^{[49]}$, the result ${ }^{[10]}$ may be used for the concept how $30 \mathrm{~kJ}$ laser pulses of picosecond duration may produce more than $1 \mathrm{GJ}$ energy from a solid density fuel cylinder. This is indeed subject to research about numerous further details of the reaction e.g., how instead of the assumed constant ultrahigh magnetic field, the temporal variations of the field have to be included.

Subject to clarify further details it is estimated how a power generator may be designed (Figure 6). The energy of the alpha particles can be converted with a gain above $90 \%$ electrostatically by their motion against a negative electric field (Figure 6) in the range of 1.4 MV. Heat generation is in the range of percents of the fusion energy. The mechanical shock of the alphas is reduced by the square root of the ratio of nuclear energy over chemical energy to negligible levels.

The just reported HB11 fusion gain for producing alpha particle energy of more than GJ, equal to $277 \mathrm{kWh}$ by the laser pulse 2 in Figure 3 of $30 \mathrm{~kJ}$, permits in principle the scheme of an economic and absolute clean power reactor 
(Figure 6). For a power station, the main part of the generated energy of the alpha particles from the level of a $-1.4 \mathrm{MV}$ voltage, can be converted into ${ }^{[54]}$ poly-phase alternating current as known from the megavolt-direct-current transmission line techniques described by Kanngiesser et al.$^{[55]}$ and Breuer $e t a l .{ }^{[56]}$. If the reactor works with a frequency of one shot per second, the electric current for conversion is $714 \mathrm{~A}$ averaged between each fusion reaction. With this operation at $1 \mathrm{~Hz}$, the reactor can produce estimated power of more than $\$ 300$ Million per year covering operational costs and an attractive profit.

\section{Conclusions}

A conclusion may be given with some remarks about next steps of activities. A possible 'potential to the best route to fusion energy' ${ }^{\text {[57] }}$ was drawn in Figure 6 which may refer to the here discussed combination of the

- reported anomaly of the very significant increase of the HB11 fusion gains ${ }^{[8,9]}$;

- with the measurement of ultrahigh magnetic fields ${ }^{[7]}$;

- and with the picosecond initiated ultrahigh nonthermal plasma block acceleration by the dominance of the nonlinear (ponderomotive) force predicted (Figure 1) $^{[12]}$ and measured first by Sauerbrey ${ }^{[13]}$.

The absolute rocklike confirmation of the last fact by the theoretical basis of the Maxwellian stress tensor and the experimental verification based on $\mathrm{CPA}^{[14]}$ with the first picosecond laser pulses of $2 \mathrm{PW}$ power ${ }^{[15,58,59]}$ needed a recognition in view of experimental facts from a flood of experiments about very different kinds of accelerations (including positron production and gamma rays for nuclear transmutations, etc.). It was a very rare clarification from a most exceptional observation of $\mathrm{x}$-ray emission by Zhang et al. ${ }^{[23]}$ why the measurements of Sauerbrey ${ }^{[13]}$ and later ${ }^{[18,19]}$, etc. were so dramatically unique: one had to avoid relativistic self-focusing by using laser pulses of extreme contrast ratios. As a point of next research, these conditions for the plasma block generation (also for hadron cancer therapy ${ }^{[30]}$ ) should explore the final limits of the conditions of the experiments first verified by Sauerbrey ${ }^{[13]}$.

Another need for experimental exploration is the numerically very detailed picosecond initiation of the fusion reactions in solid density fuel by Chu and Bobin ${ }^{[32,33]}$ confirmed by alternative computation codes and conditions. As an experiment in this direction was the exceptionally high fusion gains measured by Norreys et al. ${ }^{[60]}$ as discussed in connection to the present topic ${ }^{[22]}$. These experimental conditions were close to the irradiation of diamond foils of few nanometers thickness ${ }^{[61]}$ where the foils were not transparent above a thickness of $3 \%$ of the wave lengths with sub-picosecond laser pulses. There was no tunneling or other classical mechanism but a proof of the dielectric explosion by the nonlinear force ${ }^{[62]}$.

Another point of exploration will be the detailed study of the cylindrical solid density fusion fuel in the axis of the coils with the $10 \mathrm{kT}$ magnetic fields (Figure 3) to see at what field the ionization of the fuel will happen and at what time the best irradiation by the laser 2 should initiate the ignition. Another point of research will be the extraordinary increased HB11 fusion gains ${ }^{[8,9,47]}$ as basis of the unique avalanche reaction. It should be mentioned that the expression 'avalanche' or 'chain' reaction was used first in the publications submitted in the same months ${ }^{[9,38]}$. Earlier, reference was given to "secondary reactions, ${ }^{\text {, }}{ }^{[4]}$ until clarification was reached in documents with the German Patent office. The consideration of the avalanche process for the results of Ref. [8] was initiated later ${ }^{[63]}$ based on Ref. [10] with reference to an IZEST conference ${ }^{[46]}$ leading to the result of Ref. [64]. Finally, the dramatic expansion of petawatt laser developments has to be motivated also for the aims of boron laser fusion ${ }^{[65,66]}$ beyond the steps to $200 \mathrm{PW}$ pulses $^{[39]}$.

\section{References}

1. H. Hora, G. Korn, L. Giuffrida, D. Margarone, A. Picciotto, J. Krasa, K. Jungwirth, J. Ullschmied, P. Lalousis, S. Eliezer, G. H. Miley, S. Moustaizis, and G. Mourou, Laser Part. Beams 33, 607 (2015).

2. M. L. E. Oliphant and L. Rutherford, Proc. R. Soc. Lond. A 141, 259 (1933).

3. T. Weaver, G. Zimmerman, and L. Wood, Exotic CTR fuel: Non-thermal effects and laser fusion application, Report UCRL-74938 (1973).

4. M. Kouhi, M. Ghoraneviss, B. Malekynia, H. Hora, G. H. Miley, A. H. Sari, N. Azizi, and S. S. Razavipour, Laser Part. Beams 29, 125 (2011).

5. H. Azechi, T. Jitsuno, T. Kanabe, M. Katayama, K. Mima, N. Miyanaga, M. Nakai, S. Nakai, H. Nakaichi, A. Nishiguchi, P. A. Norreys, Y. Setsuhara, M. Takagi, M. Yamanaka, and C. Yamanaka, Laser Part. Beams 9, 193 (1991).

6. H. Hora, G. H. Miley, M. Ghorannviss, H. Malekynia, N. Azizi, and X.-T. He, Energy Environ. Sci. 3, 479 (2010).

7. S. Fujioka, Z. Zhang, K. Ishihara, K. Shigemori, Y. Hironaka, T. Johzaki, A. Sun ahara, N. Yamamoto, H. Nakashima, T. Watanabe, H. Shiraga, H. Nishimura, and H. Azechi, Sci. Rep. 3, 1170 (2013).

8. A. Picciotto, D. Margarone, A. Velyhan, P. Bellini, J. Krasa, A. Szydlowski, G. Bertuccio, Y. Shi, A. Margarone, J. Prokupek, A. Malinowska, E. Krouski, J. Ullschmied, L. Laska, M. Kucharik, and G. Korn, Phys. Rev. X 4, 031030 (2014).

9. C. Labaune, S. Deprierraux, S. Goyon, C. Loisel, G. Yahia, and J. Rafelski, Nat. Commun. 4, 2506 (2013).

10. P. Lalousis, H. Hora, and S. Moustaizis, Laser Part. Beams 32, 409 (2014).

11. H. Hora, Plasmas at High Temperature and Densities (Springer, 1991). 
12. H. Hora, Physics of Laser Driven Plasmas (Wiley, 1981) pp. 178 and 179.

13. R. Sauerbrey, Phys. Plasmas 3, 4712 (1996).

14. D. Stickland and G. Mourou, Opt. Commun. 56, 219 (1985).

15. Barty C. P. J, M. Key, J. Britten, R. Beach, G. Beer, C. Brown, S. Bryan, J. Caird, T. Carlson, J. Crane, J. Dawson, A. C. Erlandson, D. Fittinghoff, M. Hermann, C. Hoaglan, A. Iyer, L. Jones, II, I. Jovanovic, A. Komashko, O. Landen, Z. Liao, W. Molander, S. Mitchell, E. Moses, N. Nielsen, H. H. Nguyen, J. Nissen, S. Payne, D. Pennington, L. Risinger, M. Rushford, K. Skulina, M. Spaeth, B. Stuart, G. Tietbohl, and B. Wattellier, Nucl. Fusion 44, S266 (2004).

16. H. Hora, Laser Part. Beams 27, 207 (2009).

17. P. Lalousis, H. Hora, S. Eliezer, J.-M. Martinez-Val, S. Moustaizis, G. H. Miley, and G. Mourou, Phys. Lett. A 377, 885 (2013)

18. I. B. Földes, J. S. Bakos, K. Gal, Y. Juhasz, M. A. Kedves, G. Koscis, S. Szatmari, and G. Veres, Laser Phys. 10, 264 (2000).

19. J. Badziak, J. Makowski, P. Paris, L. Ryc, J. Wolowski, E. Woryna, A. A. Kozlov, and A. B. Vankov, Laser Part. Beams 17, 313 (1999)

20. H. Hora, J. Badziak, M. N. Read, Y. T. Li, T. J. Liang, H. Liu, Z. M. Shang, J. Zhang, F. Osman, G. H. Miley, W. Y. Zhang, X. T. He, H. S. Peng, S. Glowacz, S. Jablonski, J. Wolowski, Z. Skladanowski, K. Jungwirth, K. Rohlena, and J. Ullschmied, Phys. Plasmas 14, 072701 (2007).

21. H. Hora, J. Badziak, F. P. Boody, R. Höpfl, K. Jungwirth, B. Kralikowa, J. Kraska, L. Laska, P. Parys, V. Perina, M. Pfeifer, K. Rohlena, J. Skala, J. Ullschmied, J. Wolowski, and E. Woryna, Opt. Commun. 207, 333 (2002).

22. H. Hora, Czech. J. Phys. 53, 199 (2003).

23. M. Zhang, J. T. He, D. B. Chen, Z. H. Li, Y. Zhang, L. Wong, Z. H. B. F. Feng, D. F. Zhang, X. W. Tang, and J. Zhang, Phys. Rev. E 57, 3745 (1998).

24. U. Teubner, I. Uschmann, P. Gibbon, D. Altenbernd, E. Förster, T. Feurer, R. Sauerbrey, G. Hirst, M. H. Key, J. Lister, and D. Neely, Phys. Rev. E 54, 4167 (1996).

25. X. Yang, G. H. Miley, K. A. Flippo, and H. Hora, Phys. Plasmas 18, 032703 (2011).

26. D. Neely, in 2nd International Symposium on High Power Laser Science and Engineering (2016), p. 87.

27. H. Liu, Y. Li, and D. Neely, in 2nd International Symposium on High Power Laser Science and Engineering (2016), p. 126.

28. B. M. Hegelich, in 2nd International Symposium on High Power Laser Science and Engineering (2016), p. 38.

29. S. A. Gaillard, T. Kluge, K. A. Flippo, M. Bussmann, B. Gall, T. Lockard, M. Geissel, D. T. Offermann, M. Schollmeier, Y. Sentoku, and T. E. Cowan, Phys. Plasmas 18, 056710 (2011).

30. R. Banati, H. Hora, P. Lalousis, and S. Moustaizis, J. Intense Pulsed Lasers Appl. Adv. Phys. 4, 11 (2014).

31. H. Hora and R. Banati, in 1st Symposium Romanian Embassy (2015).

32. M. S. Chu, Phys. Fluids 15, 412 (1972).

33. J.-L. Bobin, in Laser Interaction and Related Plasma Phenomena, H. Schwarz and H. Hora (eds), Vol. 3B (Plenum Press, 1974), p. 465.

34. H. Hora, B. Malekynia, M. Ghoranneviss, G. H. Miley, and X.-T. He, Appl. Phys. Lett. 93, 011101 (2008).

35. P. Lalousis and H. Hora, Laser Part. Beams 1, 283 (1983).

36. H. Hora, P. Lalousis, and S. Eliezer, Phys. Rev. Lett. 53, 1650 (1984).

37. H. Hora, G. H. Miley, M. Ghoranneviss, B. Malekynia, and N. Azizi, Opt. Commun. 282, 4124 (2009).
38. H. Hora, P. Lalousis, and S. Moustaizis, Laser Part. Beams 32, 63 (2014)

39. C. P. J. Barty, J. Phys. Conf. 717, 012086 (2016).

40. H. Hora, Laser Plasma Physics - Forces and the Nonlinearity Principle 2nd edn. (SPIE, 2016), chapter 10.4.

41. H. Hora, P. Lalousis, S. Moustaizis, I. Földes, G. H. Miley, X. Yang, X. T. He, S. Eliezer, and J.-M. Martinez-Val, in IAEA Proceed. Fusion Energy (2012), paper IFE/P6-03 http://www.naweb.iaea.org/napc/physics/FEC/FEC2012/pape rs/27_IFEP603.pdf.

42. V. S. Belyaev, A. P. Matafonov, V. I. Vinogradov, V. P. Krainov, V. S. Lisitsa, A. S. Roussetski, G. N. Ignatyev, and V. P. Andrianov, Phys. Rev. E 72, 026406 (2005).

43. W. Rebentisch, see P. L. Günther, Paul Sessle \& W. Rebentisch, Z. Anorg. Allg. Chem., 250, 357 (1943).

44. W. Shockly, Electrons and Holes in Semiconductors (Van Norstrand, 1952)

45. B. W. Boreham, D. S. Newman, R. Höpfl, and H. Hora, J. Appl. Phys. 78, 5848 (1995).

46. G. Korn, D. Margarone, and A. Picciotto, in IZEST Conference (2014).

47. C. Labaune, C. Baccou, V. Yahla, C. Neuville, and J. Rafelski., Nat. Sci. Rep. 6, 21202 (2016).

48. W. M. Nevins and C. Swain, Nucl. Fusion 40, 865 (2000).

49. S. Eliezer, H. Hora, G. Korn, N. Nissim, and J. M. MartinezVal, Phys. Plasmas 23, 050704 (2016). A comment follows in Issue 10 (October) of same journal.

50. V. E. Fortov, I. T. Iakubov, and H. A. Bronstein, Physics of Non-ideal Plasma (World Scientific, 2000).

51. V. S. Belyaev, V. P. Krainov, A. P. Matafonov, and B. V. Zagreev, Laser Phys. Lett. 12, 096001 (2015).

52. G. H. Miley, H. Hora, and G. Kirchhoff, J. Phys. Conf. Proc. 717, 012095 (2016).

53. H. Hora and G. J. Kirchhoff, in World Patent Application WO 2015/144190 Al (2015)

54. D. J. Gotham and G. T. Heydt, IEEE Trans. Power Systems 13, 60 (1998).

55. K. W. Kanngiesser, D. H. Huang, and H. Lips, High-voltage Direct Current Transmission-Systems and Planning, Siemens Monographs Munich, EV HA 7. (1994).

56. W. Breuer, D. Povh, D. Retzmann, Ch. Urbanke, and M. Weinhold, in 20th World Energy Congress (2007).

57. S. Haan, in Nuclear Power without radioactivity Highlights in Chemical Technology (2010).

58. G. Mourou, C. P. J. Barty, and M. D. Perry, Phys. Today 51, 22 (1998).

59. T. E. Cowan, M. D. Perry, M. H. Key, T. R. Dittmire, S. P. Hatchett, E. A. Henry, J. D. Moody, M. J. Moran, D. M. Pennington, T. W. Phillips, T. C. Sangster, J. A. Sefcik, M. S. Singh, R. A. Snavely, M. A. Stoyer, S. C. Wilks, P. W. Young, Y. Takahashi, B. Dong, W. Fountain, T. Parnell, J. Johnson, A. W. Hunt, and T. Kuhl, Laser Part. Beams 17, 773 (1999).

60. P. A. Norreys, A. P. Fews, F. N. Beg, A. R. Bell, A. E. Dangor, P. Lee, M. B. Nelson, H. Schmidt, M. Tatarakis, and M. D. Cable, Plasma Phys. Control. Fusion 40, 175 (1998).

61. S. Steinke, A. Henig, M. Schnürer, T. Sokollik, P. V. Nickles, D. Jung, D. Kiefer, R. Hörlein, J. Schreiber, T. Tajima, X. Q. Yan, M. Hegelich, J. Meyer-ter-Vehn, W. Sandner, and D. Habs, Laser Part. Beams 28, 216 (2010).

62. H. Hora, Laser Part. Beams 30, 325 (2012).

63. G. Korn, D. Margarone, and A. Picciotto, in 2014 BoronProton Nuclear Fusion Enhancement Induced in Boron-doped Silicon Targets by Low-contrast Pulsed Lasers (2014). 
64. H. Hora, P. Lalousis, L. Giuffrida, D. Margarone, G. Korn, S. Eliezer, and G. H. Miley, SPIE Proc. 9515, 951518 (2015). 65. C. Danson, et al., High Power Lasers Sci. Engng 3, 38 (2015).
66. R. X. Li, X. Liang, Z. Gan, H. Lu, L. Yu, D. Yin, Y. Leng, $\mathrm{X}$. Lu, C. Wang, and $\mathrm{Z}$. $\mathrm{Xu}$, in 4th Advanced Lasers and Photon Sources (ALPS'15) (2015). 